\title{
Comparison of HRCT Chest and RT-PCR in Diagnosis of COVID-19
}

\author{
Nadia Hanif ${ }^{1}$, Ghazala Rubi ${ }^{2}$, Nadia Irshad ${ }^{1}$, Saima Ameer ${ }^{1}$, Uzma Habib ${ }^{1}$ and Syed Razi Haider Zaidi ${ }^{3}$ \\ ${ }^{1}$ Department of Radiology, PGMI / AMC / Lahore General Hospital, Lahore, Pakistan \\ ${ }^{2}$ Department of Central Research Lab, PGMI / AMC / Lahore General Hospital, Lahore, Pakistan \\ ${ }^{3}$ Department of Community Medicine, PGMI / AMC / Lahore General Hospital, Lahore, Pakistan
}

\begin{abstract}
Objective: To compare the diagnostic accuracies of HRCT chest and RT-PCR results in diagnosis of coronavirus disease (COVID-19) in a tertiary care hospital in Lahore.

Study Design: Descriptive study.

Place and Duration of Study: Department of Radiology and Central Research Lab, Lahore General Hospital, Lahore, from April to July, 2020.

Methodology: Patients aged 18 to 83 years, who had clinically suspected symptoms of COVID-19 (fever, cough/sore throat or shortness of breath) presenting in outpatient or emergency department, were included. These patients had their HRCT chest conducted from Radiology Department and RT-PCR performed at Central Research Lab. These data were retrieved from electronic system of PACS. Results were categorised into positive and negative findings for COVID-19. Diagnostic accuracies of HRCT chest and first RT-PCR along with $95 \%$ confidence interval were calculated.

Results: A total of 94 patients, 55 (58.5\%) males and 39 (41.5\%) were females. Out of them, $83 \%$ patients had positive HRCT chest findings of COVID-19, $17 \%$ had negative HRCT chest findings; while $40.4 \%$ had positive and $59.6 \%$ had negative first PCR. Among the repeat second PCR, $19.6 \%$ had negative, $1.8 \%$ had positive PCR results; while $78.6 \%$ patients didn't undergo repeat PCR. The sensitivity, specificity, NPV, PPV and accuracy of HRCT chest was $92 \%, 23 \%, 81 \%, 45 \%$, and $51 \%$; while of first RT-PCR was $45 \%, 81 \%$, $23 \%, 92 \%$ and $51 \%$, respectively.
\end{abstract}

Conclusion: The sensitivity of HRCT chest is higher (92\%) as compared to first RT-PCR (45\%).

Key Words: COVID-19, RT-PCR, HRCT chest, Sensitivity, Specificity.

How to cite this article: Hanif N, Rubi G, Irshad N, Ameer S, Habib U, Zaidi SRH. Comparison of HRCT Chest and RT-PCR in Diagnosis of COVID-19. J Coll Physicians Surg Pak 2021; 31(JCPSPCR):S1-CR6.

\section{INTRODUCTION}

Coronavirus disease (COVID-19) is caused by severe respiratory syndrome corona virus (SARS-COV-2), which emerged from the city of Wuhan, China. Initially through foreign, followed by local transmission of this disease, COVID-19 spread as a pandemic including almost 213 countries with America, Italy, Spain, UK being affected the most. Pakistan was amongst the top 20 of the most affected countries, where the first case was reported in February, 2020 in Karachi. ${ }^{1}$ According to COVID-19 Health Advisory Platform by Ministry of National Health Pakistan till 2nd July, 2020, there were 221,896 confirmed cases with 4,551 deaths.

Correspondence to: Dr. Nadia Hanif, Department of Radiology, PGMI / AMC / Lahore General Hospital, Lahore, Pakistan

E-mail: nadiach@hotmail.com

Received: August 17, 2020; Revised: November 07, 2020; Accepted: December 01, 2020

DOI: https://doi.org/10.29271/jcpsp.2021.JCPSPCR.CR1
Although much time has passed since the outbreak of this atypical virus, no specific or confirmed prophylactic as well as curative treatment has been approved by WHO. Therefore, it has become necessary to diagnose the infected person at an early stage, so that its spread can be controlled. Healthcare workers are being increasingly affected with the novel infection ranging from $15-18 \%$ and in some cases up to $20 \%$ of the infected population. ${ }^{2}$

RT-PCR taken by nasal or oropharyngeal route is considered the reference standard for the detection of COVID-19 disease. ${ }^{3}$ Due to emerging mutant strains of SARS-COV-2, the false positive PCR results are raising, making this pandemic to spread more due to lack of early diagnosis and isolation. There are limitations like sample collection, transportation, kit performance and financial burden as well, for countries like Pakistan.

HRCT chest plays a pivotal role in diagnosing COVID-19 due to typical imaging features of bilateral, peripheral, sub-pleural, ground glass attenuation in a posterior distribution. ${ }^{4,5}$ This may progress to consolidation or crazy paving appearance ending in bilateral white out lung ${ }^{6,7}$ Xie suggested that above typical HRCT chest findings with first negative RT- PCR has necessitated the need for using HRCT as an adjuvant to PCR. ${ }^{8}$ The positive rates of RT-PCR assay and chest CT imaging are reported to be $59 \%$ 
(601/1014), and 88\% (888/1014) for the diagnosis of suspected patients with COVID-19, respectively. With RT-PCR as a reference, the sensitivity of chest CT imaging for COVID-19 was $97 \%$ $(580 / 601) .^{9}$

While COVID-19 continues to plague communities and societies across the Globe, bringing life to a standstill, quest for a diagnostic modality which is rapid and considerably accurate prevails. The local data on comparison of HRCT-Chest and RTPCR in diagnosis of coronavirus disease is scarce in literature till the writing of theselines.

The aim of this study was to compare their diagnostic accuracies to generate data from Pakistani population, adding to pool of global evidence.

Table I: Frequency distribution.

\begin{tabular}{|l|l|}
\hline $\mathbf{N}=\mathbf{9 4}$ & Frequency \\
\hline Age groups & $7(7.4 \%)$ \\
\hline$<25 y$ & $37(39.4 \%)$ \\
\hline $25-45 y$ & $40(42.6 \%)$ \\
\hline $45-65 y$ & $10(10.6 \%)$ \\
\hline$>65 y$ & $19(20.2 \%)$ \\
\hline Contact history & $42(44.7 \%)$ \\
\hline Positive & $33(35.1 \%)$ \\
\hline Negative & \\
\hline Unsure & $78(83.0 \%)$ \\
\hline HRCT chest & $16(17 \%)$ \\
\hline Positive & $76(97.4 \%)$ \\
\hline Negative & $2(2.6 \%)$ \\
\hline Bilateral findings & \\
\hline Unilateral findings & \\
\hline
\end{tabular}

\section{METHODOLOGY}

This was a retrospective study approved by Ethical Review Committee of Lahore General Hospital. From April to July 2020, a total of 303 CT chest were performed in this hospital; out of which, 94 were suspected of COVID -19, remaining 209 patients were suspected for other pathologies. Patients aged 18 to 83 years, irrespective of gender, who were clinically suspected of COVID-19 with at least one RT-PCR test and HRCT chest done, individuals with close contact history and admitted patients as a part of preoperative workup, were included. Patients with interval of more than seven days between HRCT chest acquisition and first PCR sampling, patients with pulmonary and extra pulmonary lung malignancy, chest trauma, previous chest surgery and pediatric age group, were excluded.

For those patients with negative first PCR but suspicious clinical symptoms suggesting COVID-19, a second PCR was conducted within 3 days after the first, the result of which was taken as diagnostic gold standard. ${ }^{10}$ For patients with only a single RT-PCR test, the testresult was taken as the diagnostic gold standard.

Real-time RT-PCR assay for detection of SARS-CoV-2 was performed by RNA extraction using Sanstach viral RNA mini kit, which analysed the qualitative dual target detection of novel COV-19ORF genes done by Systeq on RT-PCR machine with internal and external positive controls.
All the suspected patients underwent thin multislice (128 slice) spiral CT scanning covering region from thoracic inlet to inferior levels of costo-phrenic angles in a supine position. CT acquisitions were performed during a deep inspiratory breath hold without contrast administration with tube voltage $120 \mathrm{kV}$; tube current regulated by an automatic exposure control system (450 $\mathrm{mAs}$ ) and pitch of 1.4. The total scan time was 3.89 seconds with rotation time of 0.5 seconds and delay of 5 seconds with slice thickness of $1 \mathrm{~mm}$ and FOV of $406 \mathrm{~mm}$. Images were transmitted to workstation and picture archiving and communication systems (PACS) for multi planar reconstruction (MPR) and post processing. HRCT images and PCR results were then retrospectively collected. HRCT images were reviewed independently by two radiologists with nine and six years of experience in radiology, respectively, who were blinded to RT-PCR results.

Positive HRCT chest findings for COVID-19 were defined as bilateral, multifocal, multilobar ground glass opacities with or without sub-segmental consolidations or crazy paving pattern in a peripheral distribution. ${ }^{11,12}$ Negative HRCT chest findings were defined as presence of isolated lobar consolidation, pleural effusion, nodularity and absence of the positive findings of COVID-19. Indeterminate HRCT cases were defined as having multilobar ground glass opacities or consolidation with central or diffuse distribution lacking subpleural pattern or unilateral ground glass opacities. These cases were further categorised as positive and negative for COVID-19 on the basis of clinical history, mutual consensus and RT-PCR results, if available. These definitions are in accordance with the Radiological Society of North America Expert Consensus Statement on Reporting Chest CT Findings Related to COVID-19. ${ }^{13}$

The collected data was entered into SPSS version 23 and analysed through its statistical programme. Quantitative variable was presented asmean orstandard deviation, i.e. age; while qualitative variables including contact history, HRCT findings and PCR results were shown as frequencies and percentages. Diagnostic accuracies, sensitivity, specificity, positive predictive value, negative predictive value of HRCT chest and RT-PCR were calculated by constructing $2 \times 2$ frequency table. Ninety-five percent confidence interval was also calculated.

\section{RESULTS}

A total of 303 CT chest were interpreted, 209 were excluded (38 pediatric age group, 33 tuberculosis, 18 primary or secondary lung malignancy, 19 extra pulmonary malignancy, 17 trauma causes, 84 were miscellaneous category of lung collapse, empyema, lobar consolidation with air bronchogram and interstitiallung disease).

Ninety-four patients met the inclusion criteria; out of which, 55 $(58.5 \%)$ were males and 39 (41.5\%) were females. The mean age was $46.54 \pm 15.22$ years ranging from $18-83$ years. The majority of patients were in the age group of 45-65 years. Contact history with COVID-19 was positive only in $20.2 \%$ of patients (Tablel). 
Table II: Diagnostic accuracy of HRCT chest and first RT-PCR in general and on basis of gender and age groups.

\begin{tabular}{|c|c|c|c|c|c|c|c|c|c|c|}
\hline & TP & TN & FP & FN & & Sensitivity & Specificity & PPV & NPV & Accuracy \\
\hline \multirow{2}{*}{ HRCT Chest } & \multirow{2}{*}{35} & \multirow{2}{*}{13} & \multirow{2}{*}{43} & \multirow{2}{*}{3} & Percentage & $92 \%$ & $23 \%$ & $45 \%$ & $81 \%$ & $51 \%$ \\
\hline & & & & & $95 \% \mathrm{Cl}$ & $79 \%-98 \%$ & $13 \%-36 \%$ & $41 \%-49 \%$ & $57 \%-93 \%$ & $41 \%-62 \%$ \\
\hline \multirow{2}{*}{$1^{\text {st }} \mathrm{RT}-\mathrm{PCR}$} & \multirow{2}{*}{35} & \multirow{2}{*}{13} & \multirow{2}{*}{3} & \multirow{2}{*}{43} & Percentage & $45 \%$ & $81 \%$ & $92 \%$ & $23 \%$ & $51 \%$ \\
\hline & & & & & $95 \% \mathrm{Cl}$ & $34 \%-57 \%$ & $54 \%-96 \%$ & $80 \%-97 \%$ & $18 \%-29 \%$ & $41 \%-62 \%$ \\
\hline \multirow{2}{*}{$\begin{array}{l}\text { HRCT chest in male } \\
\text { population }\end{array}$} & \multirow{2}{*}{22} & \multirow{2}{*}{8} & \multirow{2}{*}{25} & \multirow{2}{*}{0} & Percentage & $100 \%$ & $24 \%$ & $47 \%$ & $100.00 \%$ & $55 \%$ \\
\hline & & & & & $95 \% \mathrm{Cl}$ & $85 \%-100 \%$ & $11 \%-42 \%$ & $42 \%-52 \%$ & & $41 \%-68 \%$ \\
\hline \multirow{2}{*}{$\begin{array}{l}\text { HRCT chest in female } \\
\text { population }\end{array}$} & \multirow{2}{*}{14} & \multirow{2}{*}{4} & \multirow{2}{*}{19} & \multirow{2}{*}{2} & Percentage & $88 \%$ & $17 \%$ & $42 \%$ & $67 \%$ & $46 \%$ \\
\hline & & & & & $95 \% \mathrm{Cl}$ & $62 \%-98 \%$ & $5 \%-39 \%$ & $36 \%-49 \%$ & $29 \%-91 \%$ & $30 \%-63 \%$ \\
\hline \multirow{2}{*}{$\begin{array}{l}\text { HRCT chest in Age } \\
<50 \text { yrs. }\end{array}$} & \multirow{2}{*}{20} & \multirow{2}{*}{12} & \multirow{2}{*}{27} & \multirow{2}{*}{1} & Percentage & $95 \%$ & $31 \%$ & $43 \%$ & $92 \%$ & $53 \%$ \\
\hline & & & & & $95 \% \mathrm{Cl}$ & $76 \%-100 \%$ & $17 \%-48 \%$ & $37 . \%-48 \%$ & $63 \%-99 \%$ & $40 \%-66 \%$ \\
\hline \multirow{2}{*}{$\begin{array}{l}\text { HRCT chest in Age } \\
>50 \text { years. }\end{array}$} & \multirow{2}{*}{16} & \multirow{2}{*}{0} & \multirow{2}{*}{17} & \multirow{2}{*}{1} & Percentage & $94 \%$ & $0 \%$ & $48 \%$ & 0 & $47 \%$ \\
\hline & & & & & $95 \% \mathrm{Cl}$ & $71 \%-100 \%$ & $0 \%-20 \%$ & $46 \%-51 \%$ & & $30 \%-65 \%$ \\
\hline
\end{tabular}

\section{$95 \% \mathrm{Cl}=95 \%$ confident interval, $P P V=P$ ositive predictive value, $N P V=$ Negative predictive $v a$}

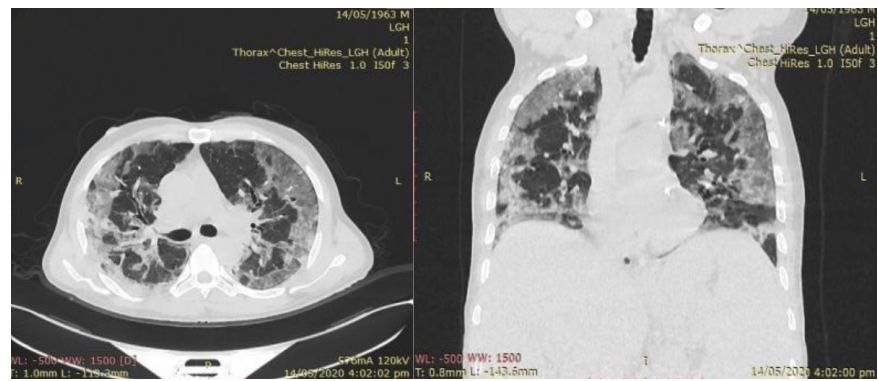

Figure 1: Axial and Coronal HRCT chest of 57 years male which shows bilateral ground glass opacities in peripheral (anterior and posterior) lung fields involving almost all lobes. This patient had negative first PCR, but CT findings suggested COVID-19 pneumonia.

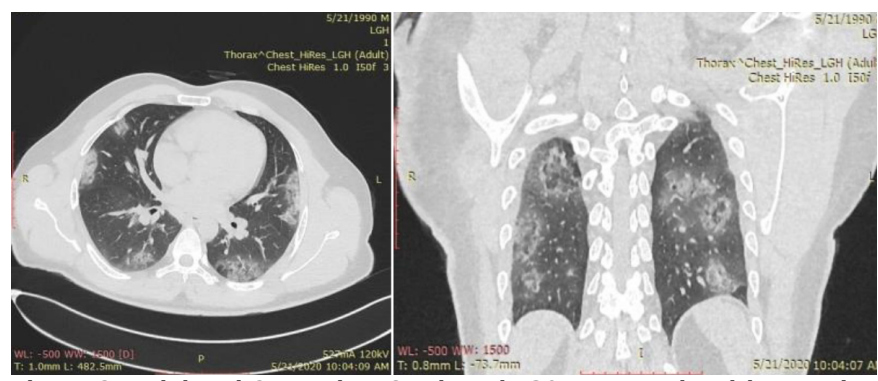

Figure 2: Axial and Coronal HRCT chest in $\mathbf{3 0}$ years male with negative first and second RT-PCR, which shows typical subpleural ground glass opacities in bilateral lung fields more marked in posterior distribution highly probable for COVID-19. Coronal image also exhibiting reverse halo pattern of GGO.

On analysis, $78 / 94(83.0 \%)$ of the total 94 patients had positive HRCT chest findings for COVID-19, while 16/94 (17\%) had negative HRCT chest findings. On contrary, only 38/94 (40.4\%) showed positive first RT-PCR results and higher number 56/94 (59.6\%) of patients had negative first PCR. There were 35/38 patients true positive having positive first RT-PCR results and typical HRCT findings of COVID -19, while three patients were false positive having positive first RT-PCR and normal HRCT. Out of 56 first PCR negative patients, $43(76.8 \%)$ were false positive with positive HRCT chest findings and 13 (23.2\%) were true negative having negative HRCT chest findings (Figure 3).

Regarding pattern of CT findings, most frequent pattern was ground glass attenuation $(72 / 78,92.3 \%)$ with bilateral distribution $(76 / 78,97.4 \%)$. The sensitivity of HRCT chest is given in Table II with confidence interval distribution.
Among males, HRCT showed sensitivity and NPV of $100 \%$ which was greater than females. Similarly, the sensitivity of HRCT in age group $>50$ years was $94 \%$ which was slightly greater when compared with sensitivity of $90 \%$ among age group $<50$ years.

Among the 56/94 (59.6\%) patients that were initially missed in first PCR, only $12 / 56(21.4 \%)$ underwent a second PCR (within 3 days of first sample). Out of these 12 cases, 11 cases (19.6\%) were declared PCR negative and only one (1.8\%) case was proven positive by second RT-PCR. Majority $(44 / 56,78.6 \%)$ patients did not undergo second RT-PCR (Figure 1 and 2).

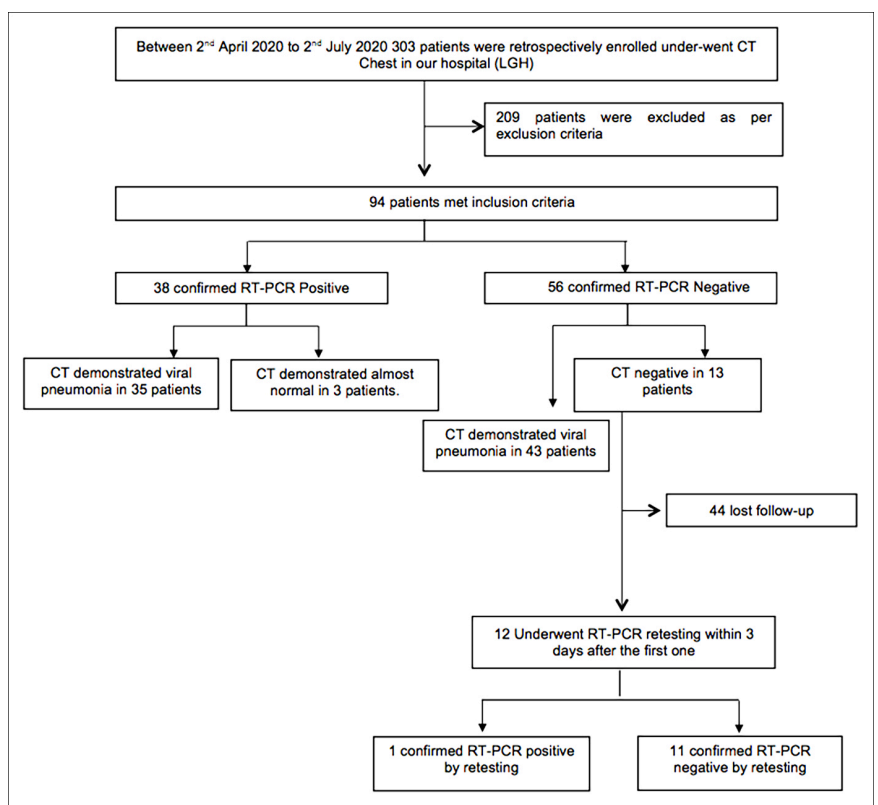

Figure 3: Flow chart of the present study.

\section{DISCUSSION}

Rapid and reliable diagnostic modality is the need of the hour for curtailing disease, both for initiating clinical management and triage as well as early quarantine which are hallmark strategies for prevention of spread. As Pakistan is one of the most affected countries by COVID-19 pandemic, knowledge of available diagnostic tools for the clinicians and preventive health experts is mandatory for early diagnosis.

Hence, HRCT chest, a non-invasive, cost effective and rapid 
diagnostic tool was evaluated with RT-PCR. The results of this study showed significant superiority of HRCT in diagnosis of COVID-19 as compared to RT-PCR, i.e. sensitivity, specificity, NPV, PPV and accuracy of HRCT chest was $92 \%, 23 \%, 81 \%$, $45 \%$, and $51 \%$; while of first RT-PCR was $45 \%, 81 \%, 23 \%$, $92 \%$ and $51 \%$, respectively. Similar results were observed by $\mathrm{Ai}$ and co-workers who conducted a study in 1,014 patients in Wuhan, China with sensitivity, specificity, NPV, PPV and diagnostic accuracy of CT chest was $97 \%, 25 \%, 68 \%, 83 \%$ and $65 \%$, respectively. Out of the 413 patients, who were tested negative in first RT-PCR, 308 (75\%) had positive CT chest findings for COVID-19. ${ }^{9}$ This study like the one by $\mathrm{Ai}$ and Dangis, showed a high negative predictive value of CT indicating low chances of person to have COVID-19 if CT features are negative for this disease and low negative predictive value of RT-PCR showing high chances of person having COVID-19 if RT-PCR results are negative. ${ }^{9,14}$

Wen et al. also reported a high sensitivity of $93 \%$ and low specificity of $53 \%$ of CT chest. ${ }^{15}$ Caruso et al. showed sensitivity, specificity, and accuracy of CT of $97 \%, 56 \%$ and $72 \%$ in a study conducted in Rome, Italy, which is comparable to the present study. ${ }^{16}$ Long also compared initial CT and PCR in a study and showed high CT sensitivity of $97.2 \%$, as compared to sensitivity of $83 \%$ of initial RT-PCR. ${ }^{17}$

Bai et al. described the radiologist performance in distinguishing COVID-19 pneumonia from other types of viral pneumonias, such as influenza, exhibiting a sensitivity ranged from 72 to $94 \%$, and specificity of $24 \%$ to $94 \%$ among three Chinese radiologists. HRCT has limited ability to differentiate COVID-19 pneumonia from other types of viral pneumonia, exhibiting a low specificity. ${ }^{18}$ This leads to more patients to be labelled as suspected COVID-19, overburdening the hospitals, healthcare providers and the healthcare facilities. But early isolation of these patients, reduced the risk of missed diagnosis of COVID-19.

In contrast, he and colleagues in their study done outside Wuhan in a group of 82 patients reported a higher sensitivity of initial RT-PCR than CT (79\% vs. 77\%).

The specificity and diagnostic accuracy of initial RT-PCR was also higher than CT (100\% vs. 96\%) and (92\% vs. $88 \%)$, respectively. They also proposed a combined strategy of using RT-PCR and CT, thus providing much higher sensitivity of $88 \%$ and diagnostic accuracies of $98 \%$. These results were different from this study due to the fact that He mostly had those patients in his study with mild symptoms who had normal chest CT, thereby reducing the sensitivity of CT. ${ }^{19}$

Song et al. described CT findings of bilateral lung involvement $(86 \%)$ with ground glass opacities $(77 \%)$ in subpleural posterior distribution mainly in lower lungs. ${ }^{20}$ Current study showed that on HRCT chest $97.4 \%$ patients had bilateral lung involvement with $92.3 \%$ having ground glass opacities. There are only two normal CT chest findings in PCR positive patients reported in this study, possibly due to early conduction of
HRCT or presence of a greater number of patients with moderate to severe disease. ${ }^{21}$

RT-PCR is believed to be reference standard for COVID-19 pneumonia, but recently sensitivity of this test is proved to be significantly low. The present results were alike Fang et al., who also reported a low sensitivity of RT-PCR as compared to CT, i.e. $71 \%$ and $98 \%$, respectively among 51 patients in China. ${ }^{22}$ There are many reasons for increase in false negative results including faulty sampling technique, improper kit performance, low patient viral load at initial stage, quality of sample collection, and sample collection from upper respiratory tract as compared to lower. ${ }^{23,24}$ PCR is relatively expensive and also takes more result processing time. Moreover, repeat PCR testing will add financial burden to the countries like Pakistan where there are already under developed healthcare facilities.

Present study showed a high percentage of individuals 56/94 $(59.6 \%)$ with negative first RT-PCR results. There were 43 patients labelled as false negative of first RT-PCR. This was a major setback of initial RT-PCR as these patients were ignored and declared as non-COVID, leading to lack of initiation of timely quarantine and early management plan. With the belief of considering first PCR as gold standard by most physicians and general public, there was non-compliance on the hand of patients and large number of patients (44/56) $78.6 \%$ lost follow-up and did not undergo second RT-PCR. Those $21.4 \%$ of patients who underwent second RT-PCR, one patient was positive and rest 11 were again negative. Li et al. also reported a high number of individuals, $63 \%$ in his study with negative first RT-PCR results, while positive rate of second RT-PCR was $12.5 \%$, high in comparison to only $1.8 \%$ in this study. ${ }^{25}$

The major limitation of this study was that serial RT-PCR tests were not available due to limited available kits in a resource-constraint environment. This study was done during pandemic period, so patients with moderate to severe symptoms were included in this study. Therefore, asymptomatic patients or those with mild symptoms were not available. The patients on ventilator support were not included in this study, as the HRCT chest were not done in these patients. Serial, follow-up CT scans were also not available in majority; as in the study setup, most were followed on conventional radiograph rather HRCT. Thus, the analysis between serial PCR and serial CT, as done by Ai et al., and Fang et al. was not possible. ${ }^{9,22}$

\section{CONCLUSION}

It is recommended that the patients with negative first RTPCR should undergo repeat PCR within three days. HRCT chest should also be done simultaneously in these patients as the typical findings of bilateral peripheral ground glass opacities, multifocal areas of sub-pleural consolidation, which are hallmark of COVID-19 are seen. HRCT is not only superior in diagnosing COVID-19, but it is also prompt and 
commonly available. Thus, it is suggested that it may be implied as first line diagnostic test at least in time of pandemic.

\section{ETHICAL APPROVAL:}

The study was approved by Ethical Review Committee of Lahore General Hospital.

\section{PATIENTS' CONSENT:}

Informed consents have been obtained from all subjects.

\section{CONFLICT OF INTEREST:}

Authors declared no conflict of interest.

\section{AUTHORS' CONTRIBUTION:}

$\mathrm{NH}$ : Conceived the idea, planned the study design, organised HRCT chest data acquisition.

GR: Organised RT-PCR data acquisition and interpretation.

$\mathrm{NI}$ : Contributed in statistical data analysis, interpretation and content writing.

SA: Participated in data interpretation and supervised the intellectual contents.

UH: Contributed in data collection and interpretation.

SRHZ: Participated in statistical analysis of data.

\section{REFERENCES}

1. Waris A, Khan AU, Ali M, Ali A, Baset A. COVID-19 outbreak: Current scenario of Pakistan. New Microbes New Infec 2020; 35:100681. doi: 10.1016/j.nmni_2020. 100681. .

2. Ali S, Noreen S, Farooq I, Bugshan A, Vohra F. Risk assessment of healthcare workers at the frontline against COVID-19. PakJ Med Sci 2020; 36(COVID19-S4):S99-S103. doi: 10.12669/pjms.36.COVID19-S4.2790.

3. Wang W, Xu Y, Gao R, Lu R, Han K, Wu G, et al. Detection of SARS-CoV-2 in different types of clinical specimens. Jama 2020; 323(18):1843-4. doi: 10.1001/jama.2020. 3786.

4. Wang Y, Dong C, Hu Y, Li C, Ren Q, Zhang X, et al. Temporal changes of $\mathrm{CT}$ findings in 90 patients with COVID-19 pneumonia: A longitudinal study. Radiology 2020; 296(2):E55-E64. doi: 10.1148/radiol.2020200843.

5. Ye Z, Zhang Y, Wang Y, Huang Z, Song B. Chest CT manifestations of new coronavirus disease 2019 (COVID-19): A pictorial review. Europ Radiol 2020; 30(8):1-9. doi: 10.1 007/s00330-020-06801-0.

6. Sohail S. Rational and practical use of imaging in COVID-19 pneumonia. PakJ Med Sci 2020; 36(COVID19S4):S130. doi: 10.12669/pjms.36.COVID19-S4.2760.

7. Xiong Y, Sun D, Liu Y, Fan Y, Zhao L, Li X, et al. Clinical and high-resolution CT features of the COVID-19 infection: Comparison of the initial and follow-up changes. Invest Radiol 2020; 55(6):332-9. .doi: 10.1097/ RLI.00000 00000000674.

8. Xie X, Zhong Z, Zhao W, Zheng C, Wang F, Liu J. Chest CT for typical 2019-nCoV pneumonia: relationship to negative RT-PCR testing. Radiology 2020; 296(2):E41-5. doi: 10.1148/radiol.2020200343.

9. Ai T, Yang Z, Hou H, Zhan C, Chen C, Lv W, et al. Correlation of chest CT and RT-PCR testing in coronavirus disease 2019 (COVID-19) in China: A report of 1014 cases. Radiology. 2020; 296(2):E32-40. doi: 10.1148/ radiol.2020 200642.

10. Kim H, Hong $\mathrm{H}$, Yoon SH. Diagnostic performance of CT and reverse transcriptase-polymerase chain reaction for coronavirus disease 2019: A meta-analysis. Radiology 2020; 296(3):E145-E55. doi: 10.1148/radiol.2020201343.

11. Han R, Huang L, Jiang $H$, Dong J, Peng $H$, Zhang D. Early clinical and CT manifestations of coronavirus disease 2019 (COVID-19) pneumonia. Am J Roentgenol 2020; 215(2):338-43. doi: 10.2214/AJR.20.22961.

12. Lee EY, Ng MY, Khong PL. COVID-19 pneumonia: What has CT taught us? Lancet Infect Dis 2020; 20(4):384-5. doi: 10.1016/S1473-3099(20)30134-1.

13. Simpson S, Kay FU, Abbara S, Bhalla S, Chung JH, Chung $M$, et al. Radiological society of North America expert consensus statement on reporting chest CT findings related to COVID-19. Endorsed by the society of thoracic radiology, the American college of radiology, and RSNA radiology. J Thoras Imaging 2020; 35(4):219-27. doi: 10.1097/RTI.0000000000000524.

14. Dangis A, Gieraerts C, Bruecker YD, Janssen L, Valgaeren $H$, Obbels $D$, et al. Accuracy and reproducibility of low-dose submillisievert chest CT for the diagnosis of COVID-19. Radiology: Cardiothoracic Imaging 2020; 2(2):e200196. doi.org/10.1148/ryct.2020200196.

15. Wen Z, Chi Y, Zhang L, Liu H, Du K, Li Z, et al. Coronavirus disease 2019: Initial detection on chest CT in a retrospective multicenter study of 103 Chinese patients. Radiology: Cardiothoracic Imaging 2020; 2(2):e200092. doi.org/ 10.1148/ ryct. 2020200092.

16. Caruso D, Zerunian M, Polici M, Pucciarelli F, Polidori T, Rucci C, et al. Chest CT features of COVID-19 in Rome, Italy. Radiol 2020; 296(2):E79-85. doi: 10.1148/radiol. 2020201237.

17. Long C, Xu H, Shen Q, Zhang X, Fan B, Wang C, et al. Diagnosis of the Coronavirus disease (COVID-19): rRT-PCR or CT? Eur J Radiol 2020; 126:108961. doi: 10.1016/j.ejrad.2020.108961.

18. Bai HX, Hsieh B, Xiong Z, Halsey K, Choi JW, Tran TM, et al. Performance of radiologists in differentiating COVID-19 from viral pneumonia on chest CT. Radiol 2020; 296(2):E46-54. doi: 10.1148/radiol.2020200823.

19. He JL, Luo L, Luo ZD, Lyu JX, Ng MY, Shen XP, et al. Diagnostic performance between CT and initial real-time RTPCR for clinically suspected 2019 coronavirus disease (COVID-19) patients outside Wuhan, China. Resp Med 2020; 168:105980. doi: 10.1016/j.rmed.2020.105980.

20. Song F, Shi N, Shan F, Zhang Z, Shen J, Lu H, et al. Emerging 2019 novel coronavirus (2019-nCoV) pneumonia. Radiol 2020; 297(3):E346. doi: 10.1148/ radiol. 2020209021.

21. Bernheim A, Mei X, Huang M, Yang Y, Fayad ZA, Zhang N, et al. Chest CT findings in coronavirus disease-19 
(COVID-19): Relationship to duration of infection. Radiology 2020; 295(3):200463. doi: 10.1148/radiol. 2020200463.

22. Fang $Y$, Zhang $H$, Xie J, Lin $M$, Ying $L$, Pang $P$, et al. Sensitivity of chest CT for COVID-19: Comparison to RT-PCR. Radiology 2020; 296(2):E115-17. doi: 10.1148/ radiol.2020200432.

23. Chan JF, Yip CC, To KK, Tang TH, Wong SC, Leung KH, et al. Improved molecular diagnosis of COVID-19 by the novel, highly sensitive and specific COVID-19-RdRp/Hel real-time reverse transcription-PCR assay validated in vitro and with clinical specimens. J Clin Microbiol 2020; 58(5):e00310-20. doi: 10.1128/JCM.00310-20.

24. Wang S, Kang B, Ma J, Zeng X, Xiao M, Guo J, et al. A deep learning algorithm using $\mathrm{CT}$ images to screen for corona virus disease (COVID-19). MedRxiv 2020; doi.10. 1101/2020.02.14.20023028.

25. Li Y, Yao L, Li J, Chen L, Song Y, Cai Z, et al. Stability issues of RT-PCR testing of SARS-CoV-2 for hospitalised patients clinically diagnosed with COVID-19. Journal of medical virology 2020; 92(7):903-8.doi: 10.1002/jmv. 25786. 